\title{
Diffusion Tensor Image Registration with Combined Tract and Tensor Features
}

\author{
Qian Wang ${ }^{1,2}$, Pew-Thian Yap ${ }^{2}$, Guorong $\mathrm{Wu}^{2}$, and Dinggang Shen ${ }^{2}$ \\ ${ }^{1}$ Department of Computer Science, University of North Carolina at Chapel Hill \\ qianwang@Cs. unc . edu \\ ${ }^{2}$ Department of Radiology and BRIC, University of North Carolina at Chapel Hill \\ \{ptyap, grwu, dgshen\} @med.unc .edu
}

\begin{abstract}
Registration of diffusion tensor (DT) images is indispensible, especially in white-matter studies involving a significant amount of data. This task is however faced with challenging issues such as the generally low SNR of diffusion-weighted images and the relatively high complexity of tensor representation. To improve the accuracy of DT image registration, we design an attribute vector that encapsulates both tract and tensor information to serve as a voxel morphological signature for effective correspondence matching. The attribute vector captures complementary information from both the global connectivity structure given by the fiber tracts and the local anatomical architecture given by the tensor regional descriptors. We incorporate this attribute vector into a multi-scale registration framework where the moving image is warped to the space of the fixed image under the guidance of tract information at a more global level (coarse scales), followed by alignment refinement using regional tensor distribution features at a more local level (fine scales). Experimental results indicate that this framework yields marked improvement over DT image registration using volumetric information alone.
\end{abstract}

\section{Introduction}

Diffusion tensor imaging (DTI) plays a vital role in the understanding of water diffusion patterns of brain tissues, providing an effective means for mapping brain structural circuitry in vivo. Water molecules are more likely to diffuse along directions tangential to the axons than directions orthogonal to the myelin sheaths. Harnessing this unique diffusion pattern allows tracing of the neuronal trajectories, and hence mapping of brain connectivity.

To make possible the comparison between individual DT images, proper spatial normalization of these images to a common space is often required. The task of DTI registration, however, is more challenging than scalar image registration. Tensors, unlike scalars, live in a space with higher dimensions that requires more complicated metrics for their effective quantification. Moreover, normalizing the DT images to a common space involves an additional step of tensor reorientation for preserving the consistency of local diffusivity pattern. Several DTI registration methods have been reported in the literature. Since scalar image registration (e.g., T1 weighted MR image) has been relatively well 
studied, a straightforward approach is to directly port state-of-the-art scalar registration algorithms for use of DT images. For example, Park et. al. [1] propose a multi-channel strategy for DTI registration by leveraging complementary information from several scalar images. In [2], tensor reorientation is explicitly incorporated in the registration optimization function. Yeo et. al. [3] utilize the Finite Strain (FS) differential for constructing a fast diffeomorphic DTI registration algorithm. For the purpose of DTI registration, various tensor similarity/dissimilarity measures have been explored [4]. Examples include mutual information [5], symmetrized Kullback-Leibler (sKL) divergence [6], and Geodesic-Loxodromes [7].

An image voxel can be represented by its attribute vector, which is essentially a vector grouping together a set of features giving descriptive and possibly distinctive information about the voxel. The effectiveness of attribute vector has been demonstrated in the registration of both scalar images and DT images. Yang et. al. [8], for instance, use prolateness, oblateness, and sphericity as the tensor attributes. In [9], a more sophisticated form of attribute vector, composing of statistical features (local mean and variance), geometrical features (edge information, and tensor principal diffusivity), is used.

Tractography, another important topic in DTI studies, streamlines fibers in the white matter (WM) and reveals neural pathways communicating individual brain regions [10]. These fibers can be grouped into bundles or tracts, each of which contains fibers with similar structural and functional characteristics. Bundles are more reproducible and consistent across subjects [11], hence suggesting their usages to guide registration of DT images. An early approach can be found in [12], where a carefully designed linear operator is iterated over the FA maps to detect bundles occurring as tubular or sheet-like structures, generating feature maps that can be used for registration using a scalar image registration method. In [13], correspondences between images are identified via tractography clustering using a polyaffine transformation to describe the deformation field.

In this paper, we design a multi-scale DTI registration framework that leverages attribute vectors for capturing both tract and tensor information. Registration is formulated as minimizing the overall difference between the attribute vectors of a pair of images. We further decompose the registration framework into two consecutive stages, each of which utilizes a different scale of features. In the first (coarse) stage, the moving image will be deformed to the fixed image under the guidance of tract derived features. Then the deformation field is transferred to the next (fine) stage and refined by using tensor related features. Details of the algorithm are provided in Section 2. Experimental results in Section 3 show that the proposed method yields improved performance in terms of registration accuracy.

\section{Method}

In the following, we will firstly brief the complete registration framework for DT images proposed in this paper (Section 2.1). Then, we will give details on the two registration stages in Section 2.2 and Section 2.3, respectively. 


\subsection{Algorithm Framework}

In tractography, fibers are streamlined by sequentially tracing the principal diffusion directions of neighboring tensors. The generated fibers can be grouped into bundles according to their topologies and anatomical properties. To register a pair of DT images, two criteria are enforced: (1) tensors with similar local features and (2) fiber tracts with identical anatomical properties should both be matched across the images. We denote the attribute vector of voxel $x$ as $\vec{a}(x)=\left[\vec{a}_{B}(x), \vec{a}_{T}(x)\right]$, where $\vec{a}_{B}(x)$ and $\vec{a}_{T}(x)$ incorporate features derived from the tracts and tensors, respectively. The elements in $\vec{a}_{B}(x)$ describe the relationship between a specific bundle and voxel $x$. In particular, each element of $\vec{a}_{B}(x)$ corresponds to a bundle and can be set if the voxel is incorporated by the bundle; otherwise it is left unset. More details about $\vec{a}_{\mathrm{B}}(\mathrm{x})$ are provided in Section 2.2. Elements of $\vec{a}_{\mathrm{T}}(\mathrm{x})$ represent the tensor features computed from $\mathrm{x}$ and its local neighborhood [9]. The registration of a fixed image $F$ and a moving image $M$ is cast as a problem of minimizing the overall distances, $D_{\mathrm{B}}(F, M \circ$ $s$ ) for tract derived features and $D_{\mathrm{T}}(F, M \circ s)$ for the tensor derived features, with a certain smooth constraint imposed on the deformation field $s$.

Multi-scale strategy is commonly used in image registration, owing to its effectiveness in avoiding local minima that often result in suboptimal solutions in high-dimensional optimization problems. Different anatomical structures are best represented at their appropriate scales. For instance, fiber bundles reflect brain connectivity and give structural information at a more global scale than an individual voxel or its adjacent neighborhood. Based on this observation, we minimize the two distances $D_{\mathrm{B}}(F, M \circ s)$ and $D_{\mathrm{T}}(F, M \circ s)$ separately at different scales, decomposing the registration problem into two consecutive stages - the tract-guided stage and the tensor-guided stage. The tract-guided stage provides a coarse estimation of deformation field for further refinement in the tensor-guided stage. A flow chart of the proposed algorithm is shown in Fig. 1. More details on the tract-guided stage and tensor-guided stage are given in Section 2.2 and Section 2.3, respectively.

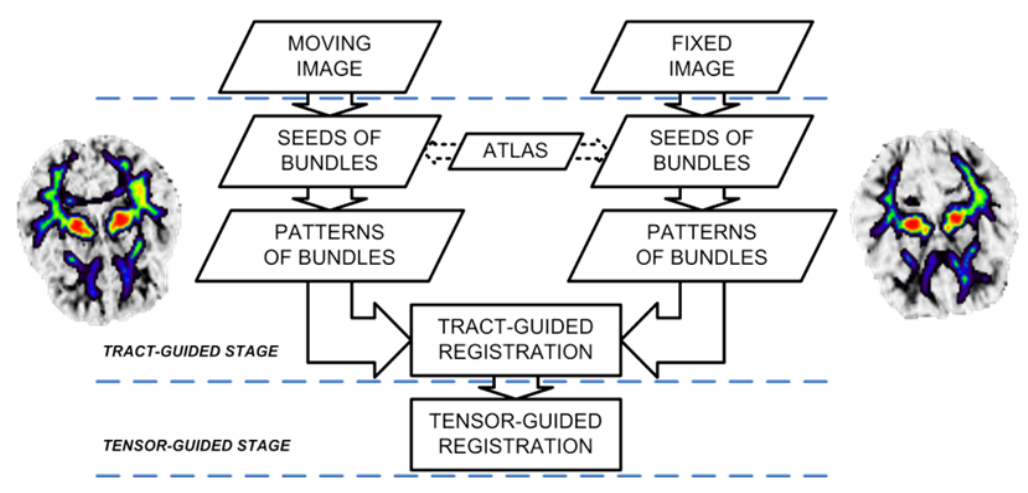

Fig. 1. The proposed DTI registration method consists of two consecutive stages: (1) tractguided registration, and (2) tensor-guided registration 


\subsection{Tract-Guided Registration}

For tract-guided registration of a pair of DT images, tractography is first applied to the images independently to yield two separate sets of fibers. After bundles are detected, the goal of registration is then to align the corresponding bundles of different images. As in Ziyan et. al. [13], bundle correspondence resulting from tractography clustering is utilized to guide DTI registration. However, spatial variation of fibers can be large, due to (1) the intrinsic complexity of brain anatomy; (2) the limited SNR in DTI scans; and (3) additional errors introduced by tractography algorithms. Especially in the case of DTI where fiber crossings cannot be modeled correctly, many fibers might be prematurely terminated. Correspondence between bundles in these situations is hence unreliable.

We take a different approach and use representations of a subset of easily identifiable tracts to help guide registration. Specifically, we have selected the corpus callosum (CC), the left/right thalamus radiata (TR), and the left/right corticospinal tracts (CST) as the driving bundles, since they traverse a large portion of the brain and represent the major WM pathways. The high reproducibility of these bundles [11] allows us to estimate their correspondences between images more reliably.

To identify the bundles, we warp the JHU WMPM [14] atlas (referred to as the "atlas" for simplicity) to the spaces of the fixed and the moving DT images by registration of the FA maps. ROIs corresponding to the selected bundles are used to determine the fiber bundles. Specifically, within the ROI for each bundle, we identify the core of the bundle by locating the voxels with fiber traversing density greater than a specified threshold. The qualified voxels form the set of bundle seeds, which we will use to generate the bundle pattern by allowing the seeds to diffuse along the underlying fibers. The bundle diffusion mechanism is explained with more details in the following. The elements of the attribute vector $\vec{a}_{B}(x)$ derived from the diffused bundle patterns are corresponding to individual bundles and used for registration.

\section{Bundle Diffusion}

Bundle diffusion is initiated at the seed voxels and proceeds along the underlying fibers. For this purpose, we have adopted the fast marching (FM) method [15]. In Fig. 2 , for instance, the fibers traversing this region (in the horizontal direction) are represented by the light blue strips. The seed region is represented by the dark red area, which is circled by the green dashed curve. In the diffusion process, the interface of the seed region propagates outward to generate the pattern $P$ after a certain amount of time, as shown in Fig. 2. For each voxel location $\mathrm{x}, P(\mathrm{x})$ records the time when the voxel is traversed by the evolving interface. At each point on the interface, the diffusion always proceeds along the surface normal. The diffusion velocity $V(\mathrm{x})$ at location $\mathrm{x}$ is calculated by integrating the total contributions of fibers passing through $\mathrm{x}$. The contribution of each fiber is equal to the cosine of the angle between the tangential direction of the fiber and the normal direction of the evolving interface. By iteratively solving the Eikonal equation $V\left\|\nabla_{\mathrm{x}} P\right\|=1$ in FM, the propagation of the region interface can be simulated numerically and the bundle pattern $P$ can thus be acquired. For corresponding tracts in both the fixed and the moving images, we allow the diffusion to last for an equal amount of time. We then reverse the sign of the 
traversing times and scale all values to the range of $[0,1]$. Hence, seed voxels have a value of 1 , which gradually decreases to zero at the stopping interface of $P$. Two real examples of the diffused bundles patterns are also shown in Fig. 1.

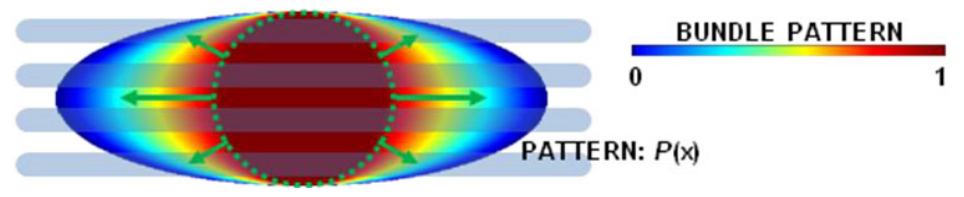

Fig. 2. Starting from the seed points within the green dashed circle, the interface propagates outward to generate the bundle pattern . Each point in the space is colored according to the interface traversing time (sign-inverted and linearly scaled to $[0,1]$ ).

\section{Bundle-Derived Attribute Vector and Registration}

To register the fixed image $F$ and the moving image $M$, we attempt to match the attribute vector $\vec{a}_{\mathrm{B}}(\mathrm{x})$ of both images. We define that the $i$-th entry of the attribute vector $\overrightarrow{\mathrm{a}}_{\mathrm{B}}(\mathrm{x})$ records the value from the diffused pattern of the $i$-th bundle. Thus the length of the attribute vector $\vec{a}_{\mathrm{B}}(\mathrm{x})$ is equal to the number of bundles used to guide registration. The term $D_{\mathrm{B}}(F, M \circ s)$ captures the overall Euclidean distance between tract-derived attribute vectors of both images, and can be minimized iteratively. In each iteration, the increment $u$ of the deformation $s$ is estimated as [16]:

$$
u=\left({ }^{t} \mathcal{T}_{\mathrm{x}} \cdot \mathcal{T}_{\mathrm{x}}+\sigma I\right)^{-1}\left(\overrightarrow{\mathrm{a}}_{\mathrm{B}}^{\mathrm{F}}(\mathrm{x})-\overrightarrow{\mathrm{a}}_{\mathrm{B}}^{\mathrm{M}}(s(\mathrm{x}))\right)^{t} \mathcal{T}_{\mathrm{x}}
$$

where $\sigma$ enforces the constraint that $u$ should be infinitesimal with respect to $s$, and the term $\mathcal{T}_{\mathrm{x}}$ (with its transpose ${ }^{t} \mathcal{T}_{\mathrm{x}}$ ) is:

$$
\mathcal{T}_{\mathrm{x}}=\frac{1}{2}\left(\nabla_{\mathrm{x}} \overrightarrow{\mathrm{a}}_{\mathrm{B}}^{\mathrm{F}}(\mathrm{x})+\nabla_{\mathrm{x}} \overrightarrow{\mathrm{a}}_{\mathrm{B}}^{\mathrm{M}}(s(\mathrm{x}))\right)
$$

Based on (1) and (2), the deformation increment $u$ is estimated to refine the deformation $s$ iteratively. The deformation $s$ is updated based on the composition rule $s \leftarrow s \circ \exp (u)$ in order to keep the generated deformation diffeomorphic. Upon estimating the deformation field, we reorient the tensors using the method described in [17]. The deformation $s$ output by the tract-guided stage here will be transferred to the following tensor-guided stage for more refinement.

\subsection{Tensor-Guided Registration}

Using tract-guided registration as initialization, further refinement to the estimated deformation $s$ is performed using tensor features. To this end, we have adopted the approach described in [9]. Specifically, tensor features, including regional features (means and variances), edge features (tensor edges and FA map edges), and geometrical features (FA values and principal diffusivities), are extracted for each voxel and incorporated into the attribute vector component $\overrightarrow{\mathrm{a}}_{\mathrm{T}}(\mathrm{x})$. A subset of voxels with distinctive attribute vectors is detected to drive the correspondence matching. 
Thin-Plate Splines (TPS) is then used to generate a dense deformation field based on the estimated displacements of the driving voxels. Unlike [9], however, only finelevel (or the highest level) registration is employed in the tensor-guided stage, since the coarse deformation is already estimated from the tract-guided stage.

\section{Experimental Results}

To demonstrate the performance of the proposed method, we performed the proposed method on both real and simulated data, and compared with DT-ITK [2] as well as F-TIMER [9] in the following.

\section{In-Vivo Dataset}

Totally, 15 DT images were acquired for our validation (Siemens Allegra scanner, $\mathrm{b}=1000 \mathrm{~s} / \mathrm{mm}^{2}$, flip angle $90^{\circ}, \quad \mathrm{TR} / \mathrm{TE}=13,640 / 82 \mathrm{~ms}$, matrix $128 \times 128$, FoV $256 \times 256 \mathrm{~mm}^{2}$, slice thickness $2 \mathrm{~mm}, 80$ contiguous slices). One image is randomly selected to be fixed, to which other 14 moving images are normalized by affine registration. We then apply all three registration methods - DT-ITK [2], F-TIMER [9], and our method - to deform the 14 images to the fixed space. For quantitative evaluation, we use the Frobenius norm to measure the distance between a pair of tensors. Specifically, we compute the tensor distance for each corresponding voxel after the moving image has been deformed to the fixed space, where tissue segmentation is available. In Table 1, the average tensor distances, as well as the respective standard deviations, of both white and grey matter voxels are listed. For white matter, our method reduces the average tensor distance by $40 \%$ over DT-ITK and $19 \%$ over F-TIMER, as $p<0.01$ in $t$-tests for both. The significantly lower tensor distance values suggest that our method is capable of DTI registration at a higher accuracy.

Table 1. The average voxelwise tensor distances

\begin{tabular}{cccc}
\hline & DT-ITK & F-TIMER & Our Method \\
\hline White Matter & $0.375 \pm 0.021$ & $0.247 \pm 0.025$ & $0.199 \pm 0.030$ \\
Grey Matter & $0.267 \pm 0.010$ & $0.219 \pm 0.031$ & $0.178 \pm 0.029$ \\
\hline
\end{tabular}

Fig. 3 shows a specific moving image in the different stages of registration to the fixed space, via both the proposed method (top) and F-TIMER (bottom). All slices are extracted from the same position and show part of cingulums. After the tract-guided registration stage in our method, the left and the right structures are apart and thus more similar to the fixed image. The output of the tract-guided stage is further refined using tensor features in our method. On the contrary, the middle level registration in F-TIMER fails to provide a good initialization as indicated by the still-connected left and right cingulums. 


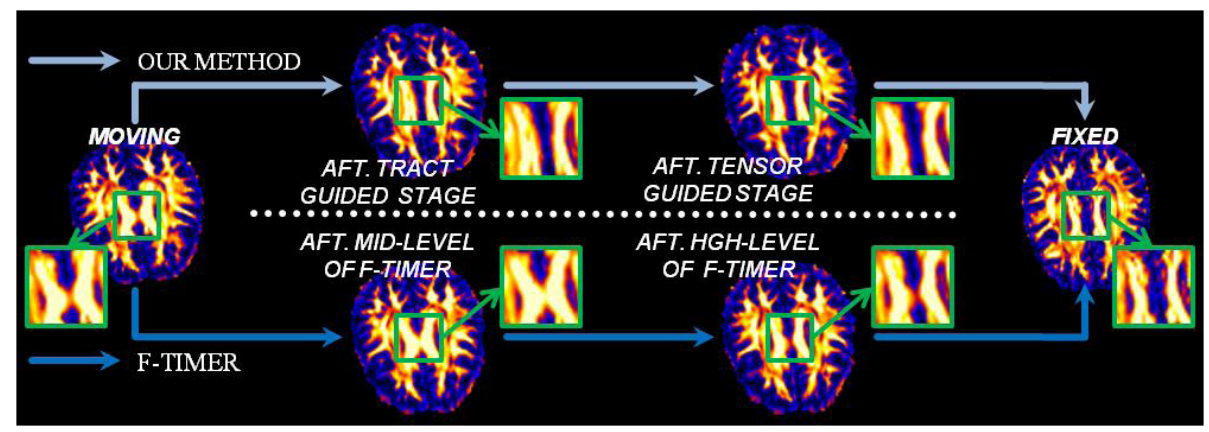

Fig. 3. The moving image is registered by both our method and F-TIMER to the fixed image. The tract-guided stage of our method yields better initialization than the middle level of FTIMER. This can be observed from the fact that the left and the right cingulums are apart using our method.

\section{Simulated Dataset}

We further evaluate the performance of the proposed method by gauging the accuracy of the estimated deformation fields. To generate a set of realistic deformation fields, we register the FA map of a randomly selected fixed image to FA maps of the other 10 images using the Demons algorithm. The estimated deformation fields are then applied to the fixed DT image to generate 10 simulated moving images. The inverse of the deformation field can hence be used as ground truth for evaluation. Across 10 simulated images, our method achieves an average deformation error of $0.637 \mathrm{~mm}$, much lower than $1.929 \mathrm{~mm}$ of DT-ITK and $0.905 \mathrm{~mm}$ of F-TIMER. The average deformation errors for the individual bundles are shown in Fig. 4, as the improvements on all bundles achieved by our method are significant statistically compared with the other two methods. It is worth noting that the deformation model in simulating data is different with any of the three methods.

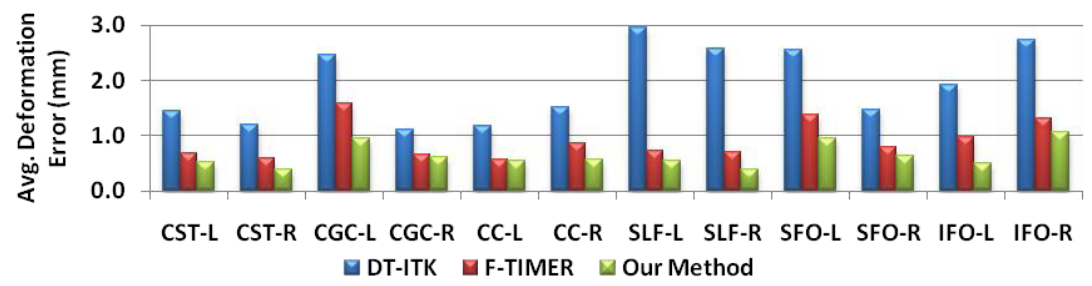

Fig. 4. The proposed method produces the lowest deformation errors in average among all three registration methods under comparison.

\section{Conclusion}

We have proposed a novel DTI registration framework by combining complementary information from both tracts and tensors. Since tract and tensor features represent 
information of different structural scales, we decompose the registration task into two consecutive stages: (1) tract-guided registration and (2) tensor-guided registration. In the tract-guided stage, attributes extracted from bundle patterns are used to give a coarse estimation of the deformation field. This aligns the major bundles and provides a good initialization for the following tensor-guided stage, where tensor features are used to refine the registration. Evaluations with both in-vivo and simulated datasets indicate that the proposed method gives superior performance when compared with DTI registration using volumetric information alone.

\section{References}

1. Park, H.-J., Kubicki, M., Shenton, M.E., Guimond, A., McCarley, R.W., Maier, S.E., Kikinis, R., Jolesz, F.A., Westin, C.-F.: Spatial normalization of diffusion tensor MRI using multiple channels. NeuroImage 20, 1995-2009 (2003)

2. Zhang, H., Yushkevich, P.A., Alexander, D.C., Gee, J.C.: Deformable registration of diffusion tensor MR images with explicit orientation optimization. Medical Image Analysis 10, 764-785 (2006)

3. Yeo, B.T.T., Vercauteren, T., Fillard, P., Pennec, X., Gotland, P., Ayache, N., Clatz, O.: DTI registration with exact finite-strain differential. In: ISBI (2008)

4. Ruiz-Alzola, J., Westin, C.F., Warfield, S.K., Alberola, C., Maier, S., Kikinis, R.: Nonrigid registration of 3D tensor medical data. Medical Image Analysis 6, 143-161 (2002)

5. Van Hecke, W., Leemans, A., D’Agostino, E., De Backer, S., Vandervliet, E., Parizel, P.M., Sijbers, J.: Nonrigid Coregistration of Diffusion Tensor Images Using a Viscous Fluid Model and Mutual Information. IEEE Trans. Medical Imaging 26, 1598-1612 (2007)

6. Chiang, M.-C., Leow, A.D., Klunder, A.D., Dutton, R.A., Barysheva, M., Rose, S.E., McMahon, K.L., de Zubicaray, G.I., Toga, A.W., Thompson, P.M.: Fluid Registration of Diffusion Tensor Images Using Information Theory. IEEE Trans. Medical Imaging 27, 442-456 (2008)

7. Irfanoglu, M.O., Machiraju, R., Sammet, S., Pierpaoli, C., Knopp, M.: Automatic Deformable Diffusion Tensor Registration for Fiber Population Analysis. In: Metaxas, D., Axel, L., Fichtinger, G., Székely, G. (eds.) MICCAI 2008, Part II. LNCS, vol. 5242, pp. 1014-1022. Springer, Heidelberg (2008)

8. Yang, J., Shen, D., Davatzikos, C., Verma, R.: Diffusion Tensor Image Registration Using Tensor Geometry and Orientation Features. In: Metaxas, D., Axel, L., Fichtinger, G., Székely, G. (eds.) MICCAI 2008, Part II. LNCS, vol. 5242, pp. 905-913. Springer, Heidelberg (2008)

9. Yap, P.T., Wu, G., Zhu, H., Lin, W., Shen, D.: F-TIMER: Fast Tensor Image Morphing for Elastic Registration. IEEE Trans. Medical Imaging 29, 1192-1203 (2010)

10. Mori, S., Zijl, P.C.M.v.: Fiber Tracking: Principles and Strategies - A Technical Review. NMR in Biomedicine 15, 468-480 (2002)

11. Wakana, S., Caprihan, A., Panzenboeck, M.M., Fallon, J.H., Perry, M., Gollub, R.L., Hua, K., Zhang, J., Jiang, H., Dubey, P., Blitz, A., van Zijl, P., Mori, S.: Reproducibility of quantitative tractography methods applied to cerebral white matter. NeuroImage 36, 630644 (2007) 
12. Goodlett, C., Davis, B., Jean, R., Gilmore, J., Gerig, G.: Improved Correspondence for DTI Population Studies Via Unbiased Atlas Building. In: Larsen, R., Nielsen, M., Sporring, J. (eds.) MICCAI 2006. LNCS, vol. 4191, pp. 260-267. Springer, Heidelberg (2006)

13. Ziyan, U., Sabuncu, M., O’Donnell, L., Westin, C.-F.: Nonlinear Registration of Diffusion MR Images Based on Fiber Bundles. In: Ayache, N., Ourselin, S., Maeder, A. (eds.) MICCAI 2007, Part I. LNCS, vol. 4791, pp. 351-358. Springer, Heidelberg (2007)

14. Mori, S., Oishi, K., Jiang, H., Jiang, L., Li, X., Akhter, K., Hua, K., Faria, A.V., Mahmood, A., Woods, R., Toga, A.W., Pike, G.B., Neto, P.R., Evans, A., Zhang, J., Huang, H., Miller, M.I., van Zijl, P., Mazziotta, J.: Stereotaxic white matter atlas based on diffusion tensor imaging in an ICBM template. NeuroImage 40, 570-582 (2008)

15. Sethian, J.A.: A Fast Marching Level Set Method for Monotonically Advancing Fronts. PNAS 93, 1591-1595 (1996)

16. Vercauteren, T., Pennec, X., Malis, E., Perchant, A., Ayache, N.: Insight into Efficient Image Registration Techniques and the Demons Algorithm. In: Karssemeijer, N., Lelieveldt, B. (eds.) IPMI 2007. LNCS, vol. 4584, pp. 495-506. Springer, Heidelberg (2007)

17. Xu, D., Mori, S., Shen, D., van Zijl, P.C.M., Davatzikos, C.: Spatial normalization of diffusion tensor fields. Magnetic Resonance in Medicine 50, 175-182 (2003) 